\title{
COHERENT STATES ASSOCIATED TO THE JACOBI GROUP - A VARIATION ON A THEME BY ERICH KÄHLER
}

\author{
STEFAN BERCEANU
}

Communicated by Ivaïlo M. Mladenov

\begin{abstract}
Using the coherent states attached to the complex Jacobi group - the semi-direct product of the Heisenberg-Weyl group with the real symplectic groupwe study some of the properties of coherent states based on the manifold which is the product of the $n$-dimensional complex plane with the Siegel upper half plane.
\end{abstract}

\section{Introduction}

In this paper we continue the investigation of the Jacobi group $[7,8,16]$ - the semidirect product of the Heisenberg-Weyl group and the symplectic group - started in $[4,5]$, using Perelomov's coherent states (CS). The Jacobi group is an important object in connection with Quantum Mechanics, Geometric Quantization, Optics, etc., $[2,9,14,15,18,20]$.

Applying the methods developed in [3], in [4] we have constructed generalized $\mathrm{CS}$ attached to the Jacobi group $G_{1}^{J}=H_{1} \rtimes \mathrm{SU}(1,1)$, based on the homogeneous Kähler manifold $\mathcal{D}_{1}^{J}=H_{1} / \mathbb{R} \times \mathrm{SU}(1,1) / \mathrm{U}(1)=\mathbb{C}^{1} \times \mathcal{D}_{1}$. Here $\mathcal{D}_{1}$ denotes the unit disk $\mathcal{D}_{1}=\{w \in \mathbb{C} ;|w|<1\}$, and $H_{n}$ is the $(2 n+1)$-dimensional real Heisenberg-Weyl group with Lie algebra $\mathfrak{h}_{n}$. In [4] we have also emphasized that, when expressed in appropriate coordinates on the manifold $\mathcal{X}_{1}^{J}=\mathbb{C} \times \mathcal{H}_{1}$, $\mathcal{H}_{1}=\{v \in \mathbb{C} ; \operatorname{Im}(v)>0\}$, the Kähler two-form $\omega_{1}$ is identical with the one considered by Kähler-Berndt [6,7,10-12].

In [5] we have considered coherent states attached to the Jacobi group $G_{n}^{J}=$ $H_{n} \rtimes \operatorname{Sp}(n, \mathbb{R})$, based on the manifold $\mathcal{D}_{n}^{J}=\mathbb{C}^{n} \times \mathcal{D}_{n}$, where $\mathcal{D}_{n}$ is the Siegel ball $\mathcal{D}_{n}=\left\{W \in M(n, \mathbb{C}) ; W=W^{t}, 1-W \bar{W}>0\right\}$. In this paper we calculate the Kähler two-form $\omega_{n}^{\prime}$ on the manifold $\mathcal{X}_{n}^{J}=\mathbb{C}^{n} \times \mathcal{H}_{n}$, where $\mathcal{H}_{n}$ is the Siegel upper half plane obtained by the Cayley transform of the Siegel ball $\mathcal{D}_{n}$. This $\omega_{n}^{\prime}$ is a " $n$ "-dimensional generalization of Kähler-Berndt's two-form $\omega_{1}^{\prime}$ on $\mathcal{X}_{1}^{J}$ to the corresponding one on $\mathcal{X}_{n}^{J}$. The physical relevance of these results follows from 
the fact that "the "gaussons" [18] can be considered as CSs indexed as the points of the manifold $\mathcal{X}_{n}^{J}$ (cf. $\S 10.1$ in the first reference [1]).

Let us recall that the denomination of "Jacobi group"-the group which realize the "squeezed states" [20] of Quantum Optics [1] - was firstly introduced by mathematicians [8]. The same group is known to physicists under other names, as the Schrödinger group [17], [5] or the "Weyl-symplectic" group [21].

The paper is laid out as follows. For self-contentedness, Section 2 recalls the basic facts established in [4] about the algebra $\mathfrak{g}_{1}^{J}$ and its holomorphic differential representation. Section 3 is devoted to a comparison of our approach in [4] with that of Kähler-Berndt. We have included in Remark 3 the differential action of the generators of the Jacobi algebra $\mathfrak{g}_{1}^{J}$ expressed in the Kähler-Berndt variables on $\mathcal{X}_{1}^{J}$. Section 4 starts recalling some facts established in [5] about holomorphic representation of the Jacobi algebra $\mathfrak{g}_{n}^{J}$. Then we present the Kähler two-form $\omega_{n}^{\prime}$ on $\mathcal{X}_{n}^{J}$, a generalization of Kähler-Berndt's construction of $\omega_{1}^{\prime}$ on $\mathcal{X}_{1}^{J}[6,7,10-13]$. These results have significance for the geometry of the manifold on which the "gaussian pure states" [18] are constructed.

\section{A Holomorphic Representation of the Jacobi Algebra $\mathfrak{g}_{1}^{J}$}

The Jacobi algebra is defined as the the semi-direct sum of the Lie algebra $\mathfrak{h}_{1}$ of the Heisenberg-Weyl Lie group and the Lie algebra of the group $\mathrm{SU}(1,1)$

$$
\mathfrak{g}_{1}^{J}:=\mathfrak{h}_{1} \rtimes \mathfrak{s u}(1,1) .
$$

The Heisenberg-Weyl algebra $\mathfrak{h}_{1}=<s 1+x a^{+}-\bar{x} a>_{s \in \mathbb{R}, x \in \mathbb{C}}$ is an ideal in $\mathfrak{g}_{1}^{J}$, determined by the commutation relations:

$$
\begin{aligned}
& {\left[a, a^{+}\right]=1} \\
& {\left[K_{0}, K_{ \pm}\right]= \pm K_{ \pm}, \quad\left[K_{-}, K_{+}\right]=2 K_{0}} \\
& {\left[a, K_{+}\right]=a^{+}, \quad\left[K_{-}, a^{+}\right]=a} \\
& {\left[K_{+}, a^{+}\right]=\left[K_{-}, a\right]=0} \\
& {\left[K_{0}, a^{+}\right]=\frac{1}{2} a^{+}, \quad\left[K_{0}, a\right]=-\frac{1}{2} a}
\end{aligned}
$$

where $a\left(a^{+}\right)$are the boson annihilation (creation) operators and $K_{0,+,-}$ are the generators of $\mathrm{SU}(1,1)$.

We impose to the cyclic vector $e_{0}$ to verify simultaneously the conditions

$$
a e_{0}=0, \quad \boldsymbol{K}_{-} e_{0}=0, \quad \boldsymbol{K}_{0} e_{0}=k e_{0}, \quad k>0, \quad 2 k=2,3, \ldots
$$


Perelomov's coherent state vectors associated to the group $G_{1}^{J}$ with Lie algebra the Jacobi algebra (1), based on the manifold $M$

$$
\begin{aligned}
M & :=H_{1} / \mathbb{R} \times \operatorname{SU}(1,1) / \mathrm{U}(1) \\
M & =\mathcal{D}_{1}^{J}:=\mathbb{C} \times \mathcal{D}_{1}
\end{aligned}
$$

are defined as

$$
e_{z, w}:=\mathrm{e}^{z a^{+}+w \boldsymbol{K}_{+}} e_{0}, \quad z, w \in \mathbb{C},|w|<1 .
$$

We summarize some of the results established in [4]

Proposition 1. The differential action of the generators (2a)-(2e) of the Jacobi algebra (1) is given by the formulas

$$
\begin{aligned}
\boldsymbol{a} & =\frac{\partial}{\partial z}, \quad \boldsymbol{a}^{+}=z+w \frac{\partial}{\partial z} \\
\mathbb{K}_{-} & =\frac{\partial}{\partial w}, \quad \mathbb{K}_{0}=k+\frac{1}{2} z \frac{\partial}{\partial z}+w \frac{\partial}{\partial w} \\
\mathbb{K}_{+} & =\frac{1}{2} z^{2}+2 k w+z w \frac{\partial}{\partial z}+w^{2} \frac{\partial}{\partial w}
\end{aligned}
$$

where $z, w \in \mathbb{C},|w|<1$.

The reproducing kernel $K\left(z, w ; \bar{z}^{\prime}, \bar{w}^{\prime}\right):=\left(e_{\bar{z}, \bar{w}}, e_{\bar{z}^{\prime}, \bar{w}^{\prime}}\right)$ is

$$
K\left(z, w ; \bar{z}^{\prime}, \bar{w}^{\prime}\right)=\left(1-w \bar{w}^{\prime}\right)^{-2 k} \exp \frac{2 \bar{z}^{\prime} z+z^{2} \bar{w}^{\prime}+\bar{z}^{\prime 2} w}{2\left(1-w \bar{w}^{\prime}\right)} .
$$

The Kähler two-form $\omega_{1}$ is given by the formula

$$
-\mathrm{i} \omega_{1}=\frac{2 k}{(1-w \bar{w})^{2}} \mathrm{~d} w \wedge \mathrm{d} \bar{w}+\frac{A \wedge \bar{A}}{1-w \bar{w}}, A=\mathrm{d} z+\bar{\alpha}_{0} \mathrm{~d} w, \alpha_{0}=\frac{z+\bar{z} w}{1-w \bar{w}} .
$$

\section{Kähler-Berndt's Construction for $\mathbb{C} \times \mathcal{H}_{1}$}

Rolf Berndt has studied the real Jacobi group $G^{J}(\mathbb{R})[6,7]$. The Jacobi group appears in the context of the so called Poincaré group or The New Poincaré group the double cover of the de Sitter group $\mathrm{SO}_{0}(4,1)$ - investigated by Erich Kähler as the ten-dimensional group $G^{K}$ which invariates a hyperbolic metric [10-12]. Kähler and Berndt have investigated the Jacobi group $G_{0}^{J}(\mathbb{R}):=\mathrm{SL}_{2}(\mathbb{R}) \ltimes \mathbb{R}^{2}$ acting on the manifold $\mathcal{X}_{1}^{J}$.

The main ingredient in the proof of Remark 1 below is the Iwasawa decomposition. Let us also mention that Iwasawa decomposition was largely used in applications in Optics, see e.g. [19]. 
Remark 1. The action of $G_{0}^{J}(\mathbb{R})$ on $\mathcal{X}_{1}^{J}$ is given by $(g,(v, z)) \rightarrow\left(v_{1}, z_{1}\right), g=$ $(M, l), l=\left(l_{1}, l_{2}\right) \in \mathbb{R}^{2}$, where

$$
v_{1}=\frac{a v+b}{c v+d}, \quad z_{1}=\frac{z+l_{1} v+l_{2}}{c v+d}, \quad M=\left(\begin{array}{cc}
a & b \\
c & d
\end{array}\right) \in \mathrm{SL}_{2}(\mathbb{R}) .
$$

Let us now recall that

$$
C^{-1} \mathrm{SL}_{2}(\mathbb{R}) C=\mathrm{SU}(1,1), \quad \text { where } C=\left(\begin{array}{rr}
\mathrm{i} & \mathrm{i} \\
-1 & 1
\end{array}\right) .
$$

If $M$ is given by the third equation (9), then, under the transformation (10)

$$
M_{*}=C^{-1} M C=\left(\begin{array}{cc}
\alpha & \beta \\
\bar{\beta} & \bar{\alpha}
\end{array}\right), \quad \alpha, \beta \in \mathbb{C},|\alpha|^{2}-|\beta|^{2}=1
$$

where

$$
2 \alpha=a+d+\mathrm{i}(b-c), \quad 2 \beta=a-d-\mathrm{i}(b+c) .
$$

Now we pass to the complex group $G_{\mathbb{C}}^{J}=C^{-1} G^{J}(\mathbb{R}) C$. We have

Remark 2. The action $C^{-1} G_{0}^{J}(\mathbb{R}) C$, descends on the basis to the biholomorphic map: $\check{C}^{-1}: \mathcal{X}_{1}^{J}:=\mathcal{H}_{1} \times \mathbb{C} \rightarrow \mathcal{D}_{1}^{J}:=\mathcal{D}_{1} \times \mathbb{C}$

$$
w=\frac{v-\mathrm{i}}{v+\mathrm{i}}, \quad z=\frac{2 \mathrm{i} u}{v+\mathrm{i}}, \quad w \in \mathcal{D}_{1}, v \in \mathcal{H}_{1}, z \in \mathbb{C} .
$$

When expressed in the coordinates $(v, u) \in \mathcal{X}_{1}^{J}$ which are related to the coordinates $(w, z) \in \mathcal{D}_{1}^{J}$ by the map (13) given by Remark 2, the Kähler two-form (8) is identical with the one considered by Kähler-Berndt

$$
-\mathrm{i} \omega_{1}^{\prime}=-\frac{2 k}{(\bar{v}-v)^{2}} \mathrm{~d} v \wedge \mathrm{d} \bar{v}+\frac{2}{\mathrm{i}(\bar{v}-v)} B \wedge \bar{B}, \quad B=\mathrm{d} u-\frac{u-\bar{u}}{v-\bar{v}} \mathrm{~d} v .
$$

Remark 3. When expressed in the coordinates $(v, u) \in \mathcal{X}_{1}^{J}=\mathcal{H}_{1} \times \mathbb{C}$, related with the coordinates $(w, z) \in \mathcal{D}_{1}^{J}=\mathcal{D}_{1} \times \mathbb{C}$ by (13), the differential action of the generators (2a)-(2e) of the Jacobi algebra (1), given by Lemma 1, becomes

$$
\begin{aligned}
\boldsymbol{a} & =\frac{v+\mathrm{i}}{2 \mathrm{i}} \frac{\partial}{\partial v}, \quad \boldsymbol{a}^{+}=\frac{2 \mathrm{i} u}{v+\mathrm{i}}+\frac{v-\mathrm{i}}{2 \mathrm{i}} \frac{\partial}{\partial u} \\
\mathbb{K}_{-} & =\frac{(v+\mathrm{i})^{2}}{2 \mathrm{i}} \frac{\partial}{\partial v}+\frac{v+\mathrm{i}}{2 \mathrm{i}} u \frac{\partial}{\partial u}, \quad \mathbb{K}_{0}=k+\frac{u v}{2 \mathrm{i}} \frac{\partial}{\partial u}+\frac{v^{2}+1}{2 \mathrm{i}} \frac{\partial}{\partial v} \\
\mathbb{K}_{+} & =-\frac{2 u^{2}}{(v+\mathrm{i})^{2}}+\frac{2 k(v-\mathrm{i})}{v+\mathrm{i}}+\frac{u(v-\mathrm{i})}{2 \mathrm{i}} \frac{\partial}{\partial u}+\frac{(v-\mathrm{i})^{2}}{2 \mathrm{i}} \frac{\partial}{\partial v} .
\end{aligned}
$$




\section{The Jacobi Group $G_{n}^{J}$}

The Jacobi algebra is the the semi-direct sum

$$
\begin{gathered}
\mathfrak{g}_{n}^{J}:=\mathfrak{h}_{n} \rtimes \mathfrak{s p}(n, \mathbb{R}) \\
\mathfrak{h}_{n}=\left\langle\mathrm{i} s 1+\sum_{i=1}^{n}\left(x_{i} a_{i}^{+}-\bar{x}_{i} a_{i}\right)\right\rangle_{s \in \mathbb{R}, x_{i} \in \mathbb{C}} \\
{\left[a_{i}, a_{j}^{+}\right]=\delta_{i j}, \quad\left[a_{i}, a_{j}\right]=\left[a_{i}^{+}, a_{j}^{+}\right]=0} \\
{\left[a_{k}^{+}, K_{i j}^{+}\right]=\left[a_{k}, K_{i j}^{-}\right]=0, \quad 2\left[a_{i}, K_{k j}^{+}\right]=\delta_{i k} a_{j}^{+}+\delta_{i j} a_{k}^{+}} \\
2\left[K_{i j}^{0}, a_{k}^{+}\right]=\delta_{j k} a_{i}^{+}, \quad 2\left[a_{k}, K_{i j}^{0}\right]=\delta_{i k} a_{j} \\
2\left[K_{k j}^{-}, a_{i}^{+}\right]=\delta_{i k} a_{j}+\delta_{i j} a_{k} .
\end{gathered}
$$

Above $K_{i j}^{0,+,-}$ represent the generators of the group $\operatorname{Sp}(n, \mathbb{R})$.

Perelomov's coherent state vectors associated to the group $G_{n}^{J}$ with Lie algebra the Jacobi algebra (16), based on the complex $N$-dimensional manifold $\left(N=\frac{n(n+3)}{2}\right)$

$$
\begin{aligned}
& M:=H_{n} / \mathbb{R} \times \operatorname{Sp}(n, \mathbb{R}) / \mathrm{U}(n) \\
& M=\mathcal{D}_{n}^{J}:=\mathbb{C}^{n} \times \mathcal{D}_{n}
\end{aligned}
$$

are defined as

$$
e_{z, W}=\exp (\boldsymbol{X}) e_{0}, \quad \boldsymbol{X}:=\sum_{i} z_{i} a_{i}^{+}+\sum_{i j} w_{i j} \boldsymbol{K}_{i j}^{+}, \quad z \in \mathbb{C}^{n} ; W \in \mathcal{D}_{n}
$$

The vector $e_{0}$ verify

$$
a_{i} e_{o}=0, i=1, \cdots, n, \quad \boldsymbol{K}_{i j}^{+} e_{0} \neq 0, \quad \boldsymbol{K}_{i j}^{-} e_{0}=0, \quad \boldsymbol{K}_{i j}^{0} e_{0}=\frac{k}{4} \delta_{i j} e_{0} .
$$

The scalar product of functions in the symmetric Fock space is [5]

$$
(\phi, \psi)=\Lambda_{n} \int_{z \in \mathbb{C}^{n} ; 1-W \bar{W}>0} \bar{f}_{\phi}(z, W) f_{\psi}(z, W) Q K^{-1} \mathrm{~d} z \mathrm{~d} W
$$

where

$$
\begin{gathered}
Q=\operatorname{det}(1-W \bar{W})^{-(n+2)} \\
K=\operatorname{det}(M)^{\frac{k}{2}} \exp \frac{1}{2}[2\langle z, M z\rangle+\langle W \bar{z}, M z\rangle+\langle z, M W \bar{z}\rangle] \\
M=(1-W \bar{W})^{-1}
\end{gathered}
$$




$$
\begin{gathered}
\mathrm{d} z=\prod_{i=1}^{n} \mathrm{dRe} z_{i} \mathrm{dIm} z_{i}, \quad \mathrm{~d} W=\prod_{1 \leq i \leq j \leq n} \mathrm{dRe} w_{i j} \mathrm{~d} \operatorname{Im} w_{i j} \\
\Lambda_{n}=\frac{k-3}{2 \pi^{\frac{n(n+3)}{2}}} \prod_{i=1}^{n-1} \frac{\left(\frac{k-3}{2}-n+i\right) \Gamma(k+i-2)}{\Gamma[k+2(i-n-1)]} .
\end{gathered}
$$

Comparatively with the case of the symplectic group, a shift of $p$ to $p-1 / 2$ in the normalization constant $\Lambda_{n}=\pi^{-n} J^{-1}(p)$ is obtained [5].

On the manifold $\mathcal{D}_{n}^{J}$, we have the Kähler two-form [5]

$$
\begin{gathered}
-\mathrm{i} \omega_{n}=\frac{k}{2} \operatorname{tr}(C \wedge \bar{C})+\operatorname{tr}\left(A^{t} \bar{M} \wedge \bar{A}\right) \\
A=\mathrm{d} z+\mathrm{d} W \bar{x}, \quad C=M \mathrm{~d} W, \quad M=(1-W \bar{W})^{-1} \\
x=(1-W \bar{W})^{-1}(z+W \bar{z}), \quad W \in \mathcal{D}_{n}, z \in \mathbb{C}^{n} .
\end{gathered}
$$

Now we consider the real Jacobi group $G_{n}^{J}(\mathbb{R})=\operatorname{Sp}(n, \mathbb{R}) \ltimes H_{n}(\mathbb{R})$, where $H_{n}(\mathbb{R})$ is the real Heisenberg-Weyl group of real dimension $(2 n+1)$. Let $g=$ $(M, X, k), g^{\prime}=\left(M^{\prime}, X^{\prime}, k^{\prime}\right) \in G_{n}^{J}(\mathbb{R})$, where $X=(\lambda, \mu) \in \mathbb{R}^{2 n}$ and $(X, k) \in$ $H_{n}(\mathbb{R})$. Then the composition law in $G_{n}^{J}(\mathbb{R})$ is

$$
g g^{\prime}=\left(M M^{\prime}, X M^{\prime}+X^{\prime}, k+k^{\prime}+X M^{\prime} J X^{\prime t}\right) .
$$

We shall also consider the restricted real Jacobi group $G_{n}^{J}(\mathbb{R})_{0}$, consisting only of elements of the form above, but $g=(M, X)$.

We consider also the manifold $\mathcal{X}_{n}^{J}:=\mathcal{H}_{n} \times \mathbb{R}^{2 n}$, where $\mathcal{H}_{n}$ is Siegel upper halfplane

$$
\mathcal{H}_{n}:=\left\{Z \in M(n, \mathbb{C}) ; Z=U+\mathrm{i} V, U, V \in M(n, \mathbb{R}), U^{t}=U, V^{t}=V, V>0\right\}
$$

Let us consider an element $g=(M, l)$ in $G_{n}^{J}(\mathbb{R})_{0}$, i.e.,

$$
M=\left(\begin{array}{cc}
A & B \\
C & D
\end{array}\right) \in \operatorname{Sp}(n, \mathbb{R}) l=\left(l_{1}, l_{2}\right) \in \mathbb{R}^{2 n}
$$

and $v \in \mathcal{H}_{n}, z \in \mathbb{C}^{n} \equiv \mathbb{R}^{2 n}$. Then the action of the group $G_{n}^{J}(\mathbb{R})_{0}$ on the base manifold $\mathcal{X}_{n}^{J}$ is given by the formula $(M, l) \times(v, z) \rightarrow\left(v_{1}, z_{1}\right) \in \mathcal{X}_{n}^{J}$, where

$$
v_{1}=(A v+B)(C v+D)^{-1}, \quad z_{1}=\left(z+v l_{1}^{t}+l_{2}^{t}\right)(C v+D)^{-1} .
$$

Now we consider the transformation

$$
w=(v-\mathrm{i})(v+\mathrm{i})^{-1}, \quad z=2 \mathrm{i}(v+\mathrm{i})^{-1} u
$$


of $\mathcal{X}_{n}^{J} \rightarrow \mathcal{D}_{n}^{J}$. The first equation (30) is nothing else than the linear fractional transformation (29) - the fameous "abcd" law for laser beams [14, 18] - corresponding to the matrix $M=C^{-1}$ where $C$ is the Cayley transform of the Siegel half-plane $\mathcal{H}_{n}$ into the Siegel unit ball $\mathcal{D}_{n}$

$$
A \in M(2 n, \mathbb{R}) \rightarrow A_{\mathbb{C}} \in M(2 n, \mathbb{R})_{\mathbb{C}}, \quad A_{\mathbb{C}}=C^{-1} A C, C=\left(\begin{array}{rr}
\mathrm{i} 1 & \mathrm{i} 1 \\
-1 & 1
\end{array}\right)
$$

Under the same transformation, $C^{-1} \operatorname{Sp}(n, \mathbb{R}) C \rightarrow \operatorname{Sp}(n, \mathbb{R})_{\mathbb{C}}$, and the linear fractional transformation (29) on $\mathcal{H}_{n}$ determined by a matrix (28) becomes linear fractional transformation on $\mathcal{D}_{n}$ with the matrix $C^{-1} M C$.

Under the transformation (30), the two-form (8) on $\mathcal{D}_{n}^{J}$ becomes on $\mathcal{X}_{n}^{J}$

$$
\begin{gathered}
-\mathrm{i} \omega_{n}^{\prime}=\frac{k}{2} \operatorname{tr}\left(p^{t} \wedge \bar{p}\right)+\frac{2}{\mathrm{i}} \operatorname{tr}\left(B^{t} D \wedge \bar{B}\right) \\
D=(\bar{v}-v)^{-1}, \quad p=D \mathrm{~d} v, \quad B=\mathrm{d} u-\mathrm{d} v D(\bar{u}-u) .
\end{gathered}
$$

The form (31) is a " $n$ "-dimensional generalization of Berndt-Kähler two-form (14).

\section{References}

[1] Ali S., Antoine J. and Gazeau J., Coherent States, Wavelets, and Their Generalizations, Springer, New York, 2000, P. Drummond and Z. Ficek (Eds), Quantum Squeezing, Springer, Berlin, 2004.

[2] Bacry H. and Cadilhac M., Metaplectic Group and Fourier Optics, Phys. Rev. A 23 (1981) 2533-2536.

[3] Berceanu S. and Gheorghe A., On Equations of Motion on Hermitian Symmetric Spaces, J. Math. Phys. 33 (1992) 998-1007; Berceanu S. and Boutet de Monvel L., Linear Dynamical Systems, Coherent State Manifolds, Flows and Matrix Riccati Equation, J. Math. Phys. 34 (1993) 2353-2371.

[4] Berceanu S., A Holomorphic Representation of the Jacobi Algebra, Rev. Math. Phys. 18 (2006) 163-199.

[5] Berceanu S., A Holomorphic Representation of the Semi-Direct Sum of Symplectic and Heisenberg Lie Algebras, J. Geom. Symm. Phys. 5 (2006) 5-13; Berceanu S., A Holomorphic Representation of Jacobi Algebra in Several Dimensions, arXiv: math.DG/060404381.

[6] Berndt R., Sur l'Arithmétique du corps des fonctions elliptiques de niveau $N$, In: Seminar on Number Theory, Paris 1982-83, Progr. Math. vol. 51, Birkhäuser, Boston, 1984, pp. 21-32. 
[7] Berndt R. and Schmidt R., Elements of the Representation Theory of the Jacobi Group, Progress in Mathematics, vol. 163, Birkhäuser, Basel, 1998.

[8] Eichler M. and Zagier D., The Theory of Jacobi Forms, Progress in Mathematics vol. 55, Birkhäuser, Boston, 1985.

[9] Guillemin V. and Sternberg S., Symplectic Technics in Physics, Cambridge University Press, Cambridge, 1984.

[10] Kähler E., Die Poincaré-gruppe, Rend. Sem. Mat. Fis. Milano 53 (1983) 359-390.

[11] Kähler E., The Poincaré Group, In: Clifford Algebras and their Applications in Mathematical Physics, Canterbury, 1985, NATO Adv. Sci. Inst. Ser. C Math. Phys. Sci., vol. 183, Reidel, Dordrecht, 1986, pp. 265-272.

[12] Kähler E., Raum-Zeit-Individuum, Rend. Accad. Naz. Sci. XL Mem. Mat. 5 16 (1992) 115-177.

[13] Erich Kähler: Mathematische Werke, R. Berndt and O. Riemenschneider (Eds), de Gruyter, Berlin, 2003.

[14] Kogelnik M., Imagining of Optical Mode-Resonators with Internal Lenses, Bell Sys. Tech. J. 44 (1965) 455-494.

[15] Nazarathy M. and Shamir J., Fourier Optics Described by Operator Algebra, J. Opt. Soc. Am., 70 (1980) 150-158.

[16] Neeb H., Holomorphy and Convexity in Lie Theory, de Gruyter, Berlin, 2000.

[17] Niederer U., The Maximal Kinematical Invariance Group of the Free Schrödinger Equation, Helv. Phys. Acta. 45 (1972/73) 802-810.

[18] Simon R., Sudarshan E. and Mukunda N., Gaussian Pure States in Quantum Mechanics and the Symplectic Group, Phys. Rev. A. 37 (1988) 3028-3038.

[19] Simon R. and Mukunda N., Iwasawa Decomposition for $\mathrm{SU}(1,1)$ and the Güoy Effect for Squeezed States, Opt. Commun. 95 (1993) 39-45.

[20] Stoler P., Equivalence Classes of Minimum Uncertainty Packets, Phys. Rev. D 1 (1970) 3217-3219; —, II, Phys. Rev D 4 (1971) 1925-1926.

[21] Wolf K., Integral Transforms in Science and Engineering, Plenum, New York, 1979; Geometric Optics on Phase Space, Springer, Berlin, 2004.

Stefan Berceanu

Institute for Physics and Nuclear Engineering

Department of Theoretical Physics

PO BOX MG-6, Bucharest-Magurele

ROMANIA

E-mail address: Berceanu@theory.nipne.ro 